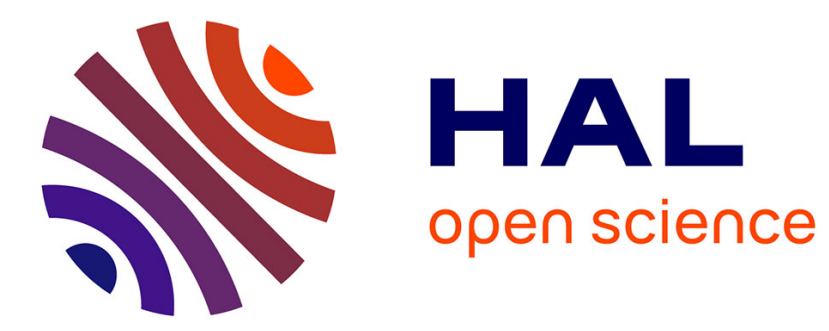

\title{
Enjeux d'une professionnalisation de la formation des enseignants de langue(s) de spécialité : exemples de l'anglais et du français de la médecine
}

\author{
Pascaline Faure
}

\section{- To cite this version:}

Pascaline Faure. Enjeux d'une professionnalisation de la formation des enseignants de langue(s) de spécialité : exemples de l'anglais et du français de la médecine. Recherche et pratiques pédagogiques en langues de spécialité - Cahiers de l'APLIUT, 2014, Vol. XXXIII Nº 1, pp.50-65. 10.4000/apliut.4170 . hal-03510341

\section{HAL Id: hal-03510341 https://hal.science/hal-03510341}

Submitted on 17 Jan 2022

HAL is a multi-disciplinary open access archive for the deposit and dissemination of scientific research documents, whether they are published or not. The documents may come from teaching and research institutions in France or abroad, or from public or private research centers.
L'archive ouverte pluridisciplinaire HAL, est destinée au dépôt et à la diffusion de documents scientifiques de niveau recherche, publiés ou non, émanant des établissements d'enseignement et de recherche français ou étrangers, des laboratoires publics ou privés. 


\title{
Enjeux d'une professionnalisation de la formation des enseignants de langue(s) de spécialité : exemples de l'anglais et du français de la médecine
}

\author{
Pascaline Faure
}

\section{Enjeux d'une professionnalisation de la formation des enseignants de langue(s) de spécialité : exemples de l'anglais et du français de la médecine}

\section{Mots-clés}

langue de la médecine, culture professionnelle, formation des enseignants, enseignement du domaine spécialisé

\section{Résumé}

La formation universitaire des enseignants qui sont conduits à enseigner une langue de spécialité en France reste très généraliste. Or, une langue de spécialité ne saurait, au vu de sa complexité, être appréhendée sans une véritable formation préalable. Dans cette contribution, nous proposons tout d'abord de rappeler que la langue de la médecine, comme d'autres langues de spécialité, est multi-référentielle et multidimensionnelle. Dans une deuxième partie, nous récapitulons les grandes étapes liminaires à la mise en place de formations en langue(s) dans le milieu de la santé et nous examinons les connaissances et les compétences requises chez l'enseignant de langue. Dans une troisième et dernière partie, nous présentons les éléments clefs qui nous paraissent nécessaires à la conception d'une formation à destination des enseignants de français et d'anglais médical, qui devrait intégrer des enseignements relatifs à la médecine et aux cultures professionnelles. Ce plaidoyer pour une professionnalisation de la formation des enseignants en LANSAD vaut pour d'autres langues de spécialité.

\author{
Issues in professionalising LSP \\ teacher education: an illustration with \\ English and French for Medical \\ Purposes
}

\section{Keywords}

medical language, professional culture, teacher education, specialised subject teaching

\begin{abstract}
In French universities, education for Languages for Specific Purposes (LSP) teachers remains highly general. Yet, due to its complexity, a specialised language cannot be mastered without prior proper training. This paper first points out that the language of medicine, like other specialised languages, is multidimensional and multireferential. In the second part, we review the major steps that should be taken prior to the implementation of teaching Languages for Medical Purposes and we examine the knowledge and skills that are required from the teacher. In the third and last part, we present key elements that we deem critical for the design of a course aimed at teachers of medical French and English, which should involve the teaching of medicine and professional cultures. This advocacy for the professionalisation of LSP teacher education applies to other specialised languages.
\end{abstract}


Depuis la mise en place du Processus de Bologne qui préconise une meilleure employabilité intra-européenne, et suite à l'introduction, dans le cadre du plan national "Réussir en licence », d'une certification en langue (le CLES ${ }^{1}$ ) au terme de la L3, le secteur LANSAD (LANgues pour Spécialistes d'Autres Disciplines) est en pleine expansion dans l'enseignement supérieur français.

Pour pouvoir assurer ces cours, les universités publient chaque année des postes d'enseignants de langue(s) dont plus de $70 \%$ sont destinés aux filières droit, médecine, sciences, économie, etc. Toutefois, cette demande exponentielle n'a pas modifié la formation des futurs enseignants de langue(s) et nombre de candidats n'ont pas le profil requis. En effet, enseigner en LANSAD requiert une bonne connaissance de la langue spécialisée qui, comme celle de la médecine, par exemple, ne saurait être réduite au seul lexique. Cette langue exige, au contraire, une formation solide qui doit pouvoir intégrer une dimension à la fois discursive, historique, culturelle, professionnelle et disciplinaire.

Dans la première partie de cet article, nous proposons de rappeler la complexité des langues de spécialité en prenant appui sur la langue de la médecine. Après l'avoir replacée dans une perspective historique, nous en énumérons certaines caractéristiques lexicales et insistons sur les cultures professionnelles qu'elle véhicule, à partir d'illustrations empruntées à l'anglo-américain et au français. La deuxième partie est consacrée à la présentation des étapes liminaires à la mise en place de formations en langue(s) de spécialité que notre expérience d'enseignantechercheuse dans le domaine de la santé nous a permis d'affiner. Dans une troisième et dernière partie, nous traitons de la question centrale de la formation des enseignants de langue(s) de spécialité à partir d'exemples de projets qui nous ont paru pertinents en anglais et en français médical.

\section{La langue de la médecine, en marge de la langue générale}

Il parait difficile de prendre toute la mesure de la complexité d'une langue de spécialité telle que la langue de la médecine sans avoir conscience de son évolution à travers les siècles. L'histoire de cette langue est en effet intimement liée à celle de la discipline qu'elle traduit, mais également à celle des acteurs qui l'utilisent et l'adaptent depuis plus de sept siècles. D'après Todorov, Mikhaïl Bakhtine écrit : «Chaque mot sent la profession, le genre [...]. Chaque mot sent le contexte et les contextes dans lesquels il a vécu sa vie sociale intense [...]»(1981: 89). La professionnalisation de la formation des enseignants de langue(s) de spécialité passe tout d'abord par un enseignement de l'histoire de cette langue, qui n'est pas forcément toujours celle de la langue générale.

\footnotetext{
${ }^{1}$ CLES : Certificat de compétences en langues de l'enseignement supérieur.
} 


\subsection{Brève histoire de la langue de la médecine}

La médecine a d'abord été écrite dans la langue grecque puisque c'est dans le monde grec qu'elle s'est développée, sous l'influence des théories d'Hippocrate au $\mathrm{V}^{\mathrm{e}}$ siècle avant J.-C. Le grec est resté la lingua franca de la médecine jusqu'à la rédaction par Celse du De Medicina, la première encyclopédie de médecine en latin, au début du $\mathrm{I}^{\mathrm{e}}$ siècle après J.-C. La conquête de l'Égypte par les Arabes en 641 a marqué un nouveau tournant dans l'histoire de la langue de la médecine. Les Arabes découvrirent la prestigieuse école d'Alexandrie, et des érudits tels que Hunayn Ibn Ishâq (809-873) et son fils Isaac Ibn Hunayn se lancèrent dans la traduction en arabe des ouvrages de médecine, dont ceux rédigés en grec par Galien. Cette tâche titanesque se prolongea jusqu'au $\mathrm{X}^{\mathrm{e}}$ siècle. Pendant ce temps, les médecins arabes tels qu'Avicenne (980-1037) et Ibn Al-Nafis (1210-1288) s'approprièrent la médecine hippocratique et l'enrichirent (voir Dachez 2012).

À leur tour, les livres d'Avicenne furent traduits de l'arabe en latin par l'italien Gérard de Cremona (1114-1187), faisant pénétrer des termes arabes dans la terminologie médicale latine. Plus tard le langage médical médiéval fut épuré de sa terminologie arabe mais un certain nombre de termes restèrent sous la forme de calques latinisés : nucha (nuque), dura mater (dure mère), pia mater (pie mère) ou encore dens sapientia (dent de sagesse).

La langue latine a régné sur la médecine jusqu'à la Renaissance puis, progressivement, à travers toute l'Europe, les enseignements ont commencé à être dispensés dans les langues nationales. Et si, dans les langues romanes comme le français, l'espagnol ou le roumain, les termes gréco-latins ont souvent été «naturalisés », d'autres langues comme les langues germaniques ont conservé la plupart des mots sous leur forme originelle ('cervelet' en français, cerebelo en espagnol et cerebelos en roumain, mais cerebellum en anglais), avec parfois quelques petites «adaptations phonétiques» (Zerebellum en allemand). $\mathrm{Ce}$ phénomène a entraîné, dans les langues germaniques et les langues slaves, la coexistence de termes gréco-latins et «nationaux »: lumbago et low back pain en anglais, Lumbago et Hexenschu $\beta$ en allemand, люмбаго еt прострел en russe.

Par la suite, la langue de la médecine s'est enrichie d'un certain nombre de termes au gré des avancées médicales en Europe. La langue de la médecine du XVIII siècle est marquée par les travaux des Français Baudelocque (forceps) et Petit (tourniquet ${ }^{2}$ ), et des Anglais Jenner (vaccination ${ }^{3}$ ) et Cullen (neurosis/ névrose). Au XIX ${ }^{\mathrm{e}}$ siècle, la langue française influence la langue médicale grâce aux travaux de Laennec (stéthoscope/stethoscope), Dupuytren (contracture de Dupuytren/ Dupuytren's contracture), Trousseau (phénomène de Trousseau/Trousseau's phenomenon), Magendie (foramen de Magendie/Magendie's foramen), Claude Bernard (glycogène/ glycogene) ou encore Sédillot (microbe/ microbe). Ce n'est qu'au lendemain de la deuxième guerre mondiale que l'anglais américain

\footnotetext{
${ }^{2}$ Un garrot. Ce terme est encore utilisé dans la langue anglaise avec le même sens.

${ }^{3}$ Le terme sera repris et élargi par Pasteur.
} 
commence à influencer la langue de la médecine. L'Europe exsangue peine à se reconstruire, et les Américains victorieux s'emparent du sceptre de la médecine, un sceptre qu'ils conservent encore de nos jours. Locked-in syndrome (1966), burnout (1970), ou encore prion ${ }^{4}$ (1982) sont des termes issus de l'anglo-américain et ont été empruntés par de nombreuses langues médicales européennes. De même, les noms des hormones et de nombreux virus sont empruntés à l'anglais : ACTH (AdrenoCorticoTropic hormone), GH (Growth Hormone), LH (Luteinizing Hormone), TSH (Thyroid-Stimulating Hormone), EBV (Epstein-Barr Virus), HPV (Human PapillomaVirus) ou CMV (CytoMegaloVirus), ainsi que les techniques récentes: drezotomie/drezotomy/drezotomia, formé de l'acronyme Dorsal Root Entry Zone et du suffixe -tomie/-tomy/-tomia précédé d'un /o/ épenthétique), TURP (TransUrethral Resection of the Prostate) et CABG (Coronary Artery Bypass Graft).

\subsection{Au-delà de la terminologie médicale}

Comme l'écrit Pierre Lerat, la langue de spécialité ne saurait être réduite à la seule terminologie :

Les langues spécialisées ont une syntaxe qui est tout à fait celle des langues de référence mais avec des prédilections en matière d'énonciation [...] et de phraséologie professionnelles (1995:28).

La langue de la médecine est également marquée par des usages particuliers. On observe, par exemple, un recours à la polysémie - essentiellement par le biais de la métaphore - à partir de mots d'usage courant : dans la langue médicale française, un 'haricot' est un bassin hygiénique et, dans la langue médicale britannique, the (operating) theater est le bloc opératoire. Certaines associations prennent un sens tout à fait particulier : en français médical, le verbe 'piquer' associé au mot 'bilan' signifie «faire un prélèvement de sang pour effectuer un dosage » et, en anglais médical, le terme domino associé à delivery est un accouchement par voie basse avec une hospitalisation de courte durée.

La langue de la médecine recourt aux ellipses : par exemple, 'Alzheimer' en français pour 'la maladie d'Alzheimer' et Alzheimer's en anglais pour Alzheimer('s) disease. De même, l'adjectif est souvent construit en hypallage (un 'coma hépatique' en français et hepatic coma en anglais renvoient à un coma secondaire à une insuffisance hépatique).

Deux autres caractéristiques majeures de la langue de la médecine sont l'abréviation (par exemple, 'cathé art' pour 'cathéter artériel' en français et appy pour appendectomy/ appendicectomy en anglais) et la siglaison : 'ACR' ('Arrêt CardioRespiratoire') en français et COPD (Chronic Obstructive Pulmonary Disease) en anglais, parfois associée au processus de dérivation lexicale : "La patiente est DCDe » (pour « décédée ») en français et «The patient ODed» (de OD overdose) en anglais (Faure 2012b).

\footnotetext{
${ }^{4}$ PRoteinaceous Infectious ONly particle.
} 
On assiste, dans la dénomination des maladies, à la multiplication des toponymes : Ebola - avec parfois la fusion de deux toponymes comme Lujo, formé à partir des villes de Lusaka en Zambie et de Johannesburg en Afrique du sud, et des orthonymes: 'insuffisance surrénalienne chronique primaire' pour la maladie d'Addison et 'sclérose latérale amyotrophique' pour la maladie de Charcot, des orthonymes qui participent au mouvement de siglaison : SLA (Faure 2012a).

Il est important également de mentionner les différents genres : article de recherche, abstract, case-report, poster, éditorial, compte-rendu d'examen, etc., qui, chacun, présente des règles de rédaction, d'organisation et de présentation bien particulières ${ }^{5}$.

\subsection{Une langue-culture}

Pour Geneviève Zarate et al, la langue est une manifestation de l'identité culturelle, et «tous les apprenants, par la langue qu'ils parlent, portent en eux les éléments visibles et invisibles d'une culture donnée » (2003 : 57). La langue de spécialité a ceci de particulier qu'elle est sous-tendue par des cultures professionnelles fortes, qui exigent que l'enseignant de langue(s) de spécialité éduque les apprenants à la diversité des pratiques médicales dans le ou les pays dont ils étudient la langue. Car si les étudiants en médecine et les médecins bénéficient ou ont bénéficié d'une formation à la pratique médicale qui inclut une approche à l'interculturel, celle-ci ne s'appuie que sur les cultures rencontrées dans le pays dans lequel la formation est dispensée. Ceci signifie que l'éducation aux cultures professionnelles « étrangères » va être une des missions de l'enseignant de langue de spécialité.

La langue de la médecine véhicule une culture professionnelle très marquée par les pratiques médicales nord-américaines, parmi lesquelles on note l'autonomisation croissante et l'augmentation des responsabilités des infirmiers (Weston 2010); le désengagement de la profession médicale face aux risques professionnels (Ofri 2013) ; le renforcement de la participation du patient dans le diagnostic et la prise en charge de sa maladie (Graham 2011) ; ou encore la responsabilisation des acteurs de santé par rapport aux dépenses de soins à travers la mise en place des dossiers médicaux personnels informatisés (DMP) et des références médicales opposables (RMO), qui indiquent les prescriptions et les actes nécessaires au traitement d'une pathologie, et le développement des médicaments génériques et de la prévention.

Pour autant, l'enseignement-apprentissage de la langue de la médecine ne saurait être réduit à la création de «grammaires » de culture et devrait inclure ce que Fred Dervin nomme «l'intersectionalité », c'est-à-dire la prise en compte de facteurs tels que la religion, le sexe et la classe sociale (Dervin 2012). En effet, en fonction de ces différents paramètres, le médecin devra parfois adopter une attitude et un discours spécifiques censés favoriser une meilleure communication et donc une prise en charge réussie (Misra-Hebert 2012). Par exemple, les questions posées au patient anglo-saxon seront généralement indirectes, et les instructions comprendront des formules de politesse «consacrées » (for me please, if you don't mind, etc.). Lors

\footnotetext{
${ }^{5}$ Voir les travaux de Didier Carnet et de Jean-Pierre Charpy.
} 
d'une consultation gynécologique aux États-Unis, afin de préserver son intimité, la patiente sera revêtue d'une blouse et placée en décubitus latéral et non dorsal comme c'est le cas en France.

Au-delà de la culture dominante, il existe des sous-cultures propres aux minorités dont il est important de tenir compte. Par exemple, les questions relatives à l'intimité ne pourront pas être posées sans que le patient afro-asiatique soit assuré, au préalable, de la confidentialité de l'entretien. Le médecin évitera de serrer la main d'une patiente du Moyen-Orient (Galanti 2008 : 6). Dans ses prescriptions, le médecin devra tenir compte du régime propre à certaines communautés (par exemple, ne pas prescrire des gélules - généralement à base de gélatine de bœuf - aux patients hindous).

La profession médicale elle-même est un exemple de sous-culture avec son jargon dont nous avons vu quelques illustrations dans la partie 1.2. - mais également avec ses valeurs, sa lecture du monde, ses codes et ses pratiques. Ainsi, dans le contexte de la médecine française, l'humour carabin (des canulars à caractère sexuel et morbide) et les rituels de «la salle de garde » (des gages de type «strip-poker», mimes sexuels, chansons paillardes sous des fresques pornographiques [Josset 2010]) participent à l'identification à la profession. Cependant, il n'est pas rare de rencontrer au sein d'un même hôpital des personnels médicaux et paramédicaux de cultures différentes. Galanti (2008: 127) souligne les différences entre les infirmières américaines, auxquelles on apprend à avoir un esprit critique (critical thinking), ce qui leur permet une plus grande autonomie, et les infirmières formées dans d'autres pays, qui n'ont pas bénéficié de cette approche et qui se considèrent plus comme des assistantes que comme des personnels autonomes.

Dans la relation médecin-patient, il est important que les différentes sous-cultures soient connues. Par exemple, au Moyen-Orient ou en Chine, le médecin fait autorité et il ne saurait voir son diagnostic remis en cause alors qu'aux États-Unis, on encourage le médecin à discuter du diagnostic avec son patient. Une méconnaissance des cultures professionnelles peut mener à des malentendus et à des conflits. Il est bon qu'un étudiant ou un professionnel de santé puisse bénéficier, dans le cadre d'un projet de mobilité, d'un enseignement des cultures professionnelles qu'il sera conduit à côtoyer ou à adopter dans la société cible.

Enfin, la culture est également didactique. En effet, la pédagogie médicale est très innovante en matière d'outils d'apprentissage : par exemple, l'APP (Apprentissage Par Problèmes), l'ARC (Apprentissage au Raisonnement Clinique), et les outils d'évaluation que sont l'ECOS (Examen Clinique Objectif Structuré), qui vise à tester des compétences dans la relation médecin-patient, l'EMS (Entrevue Médicale Simulée) et le TCS (Test de Concordance de Script), une technique d'évaluation du raisonnement clinique en contexte d'incertitude par une simulation de situations. L'enseignant de langue de spécialité peut s'appuyer sur cette culture didactique en classe et détourner les dispositifs pédagogiques qui visent initialement l'apprentissage de l'exercice médical pour proposer des tâches langagières. Nous en 
apportons quelques illustrations dans la partie 2.5.

\section{Prérequis pour concevoir une formation de langue médicale}

\subsection{Quel(s) type(s) de demande ?}

Il est important que la mise en place d'un programme de langue de spécialité corresponde à une demande précise et clairement analysée (Mangiante \& Parpette 2004). Dans le domaine de la santé, on peut identifier deux grands types de publics : celui des étudiants en formation initiale (étudiants en médecine, pharmacie, odontologie, maïeutique, soins infirmiers et médecine vétérinaire) et celui des professionnels de santé déjà engagés dans une activité professionnelle (hospitalouniversitaires, médecins, dentistes, sages-femmes, infirmiers et pharmaciens).

Selon le public, la formation vise l'acquisition de la langue de spécialité (étudiants) ou celle de la langue professionnelle (professionnels de santé). Cependant, la frontière entre les deux types de langues est très ténue, et les dénominations ellesmêmes peuvent parfois sembler floues. "Le terme Français de spécialité a été historiquement le premier à désigner des méthodes destinées à des publics spécifiques étudiant le français dans une perspective professionnelle ou universitaire » (Mangiante \& Parpette 2004 : 16). Progressivement, d'autres termes tels que Français sur objectif(s) spécifique(s) et Français langue professionnelle ont été déclinés. Selon Florence Mourlhon-Dallies (2008), ces termes sont sous-tendus par différentes visions didactiques. La langue de spécialité traduit une vision domaniale, et son mode d'organisation se fait autour des spécialités et des savoirs. La langue sur objectif spécifique est motivée par une vision occupationnelle et est organisée par compétences. La langue professionnelle traduit une vision collaborative et est axée sur un dispositif de travail ( « chaînage d'activités »). En anglais, le terme Specialised English recouvre une réalité bien différente. Apparu en $1998^{6}$ dans les programmes diffusés par la radio britannique Feba pour désigner une forme simplifiée d'anglais à destination des locuteurs non natifs, le terme n'a rien à voir avec la spécialité. En anglais, on parle de English for Specific Purposes, un terme décliné en English for Academic Purposes (EAP) et English for Occupational Purposes (EOP), deux distinctions qui permettent de différencier les plans institutionnels : l'université (EAP) et le monde professionnel (EOP).

Or, on est souvent conduit à enseigner la langue professionnelle (par exemple, dans l'enseignement de la consultation) en formation initiale et la langue de spécialité (par exemple, l'anglais de la maïeutique) en formation médicale continue afin, dans le premier cas, d'anticiper sur les situations de communication à venir du futur professionnel de santé et, dans le deuxième cas, de pallier le manque de connaissances du professionnel de santé, qui généralement, n'a pas bénéficié d'un enseignement de langue de spécialité durant ses études.

\footnotetext{
${ }^{6}$ Le terme est une adaptation de Special English, lui-même inventé en 1959 par Voice of America, un programme radiophonique américain, pour désigner un anglais volontairement simplifié.
} 
Ce qui varie en revanche, c'est le niveau linguistique de départ et les contraintes socio-professionnelles de l'apprenant, à partir desquels on détermine le nombre et la répartition des heures d'enseignement et le choix des supports (articles de vulgarisation, articles de recherche, etc.). Dans le cadre d'une formation initiale, le programme est généralement conçu comme extensif et progressif alors que les professionnels de santé vont plutôt être demandeurs d'un programme intensif.

\subsection{Quelles situations de communication ?}

Lorsque l'on doit répondre à une demande de formation en langue médicale, il est indispensable d'identifier les besoins communicationnels. Il n'est pas question, en effet, d'enseigner la langue in extenso mais de sérier les situations de communication auxquelles l'apprenant est (ou sera) confronté. Une des étapes liminaires est donc l'analyse des besoins (Chambers 1980 ; Widdowson 1983; Hutchinson \& Waters 1987) à partir d'une recension des contextes d'énonciation en formation médicale continue et des thématiques de la discipline en formation initiale.

S'agissant de formation médicale continue, il est également important de tenir compte du poste de l'apprenant dont la nature permet de déterminer celle des coénonciateurs, les types de discours utilisés et les compétences requises, telles qu'elles sont définies dans l'apprentissage par les tâches (Ellis 2003), l'approche actionnelle étant celle que nous avons choisie.

Il apparaît tout aussi important d'identifier les besoins en termes culturels, qui jouent un rôle essentiel dans les interactions Nous avons donc opté pour un modèle socioconstructiviste parce qu'il s'appuie sur les représentations de l'apprenant dont les conceptions initiales se trouvent au cœur du processus d'apprentissage. Mais nous nous appuyons également sur une approche interactionniste, empruntée à la sociologie cognitive (Bandura 1977), qui sous-tend d'ailleurs les dispositifs pédagogiques utilisés en médecine tels que le TCS.

L'enseignant de langue(s), qui ne possède généralement qu'une connaissance limitée des situations de communication, doit pouvoir accéder à des ressources qui vont lui permettre de les identifier en effectuant une observation dans un service hospitalier (en anglais, on parle de shadowing), en multipliant les contacts avec des professionnels de la discipline, ou encore en consultant des blogs à visée professionnelle ou les fiches métiers dans la langue cible.

\subsection{Quelles compétences ?}

En matière de formation en langue médicale, la recension des situations de communication permet de mettre au jour trois grands types de compétences (Faure 2012c : 115) :

- les compétences préprofessionnelles générales : lire un article (de vulgarisation ou de recherche), rédiger un abstract/une synthèse, faire une recherche documentaire, suivre une conférence et prendre des notes, et connaître l'organisation des études ; 
- les compétences professionnelles générales : rédiger un article de recherche, faire une présentation orale (communication en congrès, observation, poster, etc.), mener une interaction orale (interroger un patient, accueillir la famille, faire une transmission, etc.), rédiger un document professionnel (ordonnance, compte-rendu d'examen, courrier à un confrère, certificat, note de synthèse, etc.), connaître le système de santé et les établissements de soins ;

- les compétences professionnelles spécifiques : elles sont aussi nombreuses que les postes, les motivations personnelles (exercer dans un autre pays, obtenir un poste d'hospitalo-universitaire, faire un stage à l'étranger, etc.) et les spécialités existantes.

\subsection{Quels contenus?}

Pour être motivants et signifiants, il est important que les contenus soient choisis en lien avec la discipline, les situations de communication et les objectifs en termes d'acquisition (Mémet 2003). Dans les formations que nous dispensons en santé, nous choisissons de nous appuyer sur un contenu médical à partir duquel nous imaginons un certain nombre de tâches (compléter une planche anatomique, trouver la maladie qui correspond aux symptômes, etc.). Ce contenu médical est sélectionné parmi les thèmes étudiés par les apprenants dans la langue 1 (par exemple, la cardiologie et, plus spécifiquement, l'anatomie du cœur et les maladies cardiovasculaires). Cette approche a pour objectif une double acquisition «matière médicale/ langue de la médecine ».

En formation médicale continue, l'enseignant de langue de spécialité discute du choix des tâches et des contenus directement avec l'apprenant (par exemple, on demande à un apprenant qui souhaite travailler plus spécifiquement sur la rédaction de nous remettre des articles ou d'autres types de supports professionnels). En formation initiale, dans la mesure où les occasions d'échanger en amont de la formation sont rares, on pourra identifier les thématiques au programme de la discipline à partir des polycopiés de cours et des informations mises en ligne sur le site de la Faculté de médecine à destination des étudiants. Il s'agit là d'une façon de mettre en place un pont entre la langue et le domaine spécialisé, et d'inscrire ainsi l'enseignement de langue(s) dans une cohérence globale qui doit pouvoir favoriser l'acquisition et augmenter le degré de motivation.

Cette double approche «contenu médical/langue de la médecine » permet également à l'apprenant de totaliser ses acquis dans un contexte de manque de temps (West 1994), qu'il s'agisse de formation initiale ou continue.

\subsection{Quels outils didactiques?}

Le concept d'apprentissage par problèmes (problem-based learning en anglais) a été développé par la Faculté de médecine de 1'Université McMaster de Hamilton en Ontario dès 1970 et prend appui sur un problème pour déclencher le processus d'apprentissage à partir d'une mobilisation des connaissances acquises antérieurement, d'une réflexion et d'une mise en concurrence de plusieurs solutions ou pistes (Davis \& Harden 1999). Cette approche se prête bien à l'enseignement- 
apprentissage de la langue médicale en favorisant la réflexion et l'interaction autour de cas cliniques avec un diagnostic différentiel par exemple.

Pour André Giordan (1998), la prise en compte des conceptions de l'apprenant doit devenir le point de départ de tout projet éducatif car on n'apprend qu'à travers ce que l'on est. Par conséquent, au-delà des outils à proprement parler, c'est sur la lecture du monde de l'étudiant ou du professionnel de santé qu'il est tentant de prendre appui lorsqu'on enseigne une langue de la médecine. Parmi les sources secondaires et supports susceptibles d'être utilisés, les séries télévisées et les thrillers spécialisés permettent aux apprenants d'appréhender la profession et sa culture en adoptant une approche comparative critique (Charpy 2010). Ces supports peuvent être utilisés aussi bien dans l'enseignement-apprentissage de la grammaire (illustration du cours et énoncés constituant les exercices) que dans la pratique de la langue (jeux de rôles) (Faure 2000).

\subsection{Quel(s) impact(s) pour l'enseignant de langue ?}

Si on est en droit de considérer que le cours de langue dans le secteur LANSAD est une rencontre entre experts et que l'enseignant peut se reposer sur les connaissances « disciplinaires » des apprenants (Dudley-Evans \& St-John 1998), notre expérience d'enseignante et de formatrice nous montre qu'il est important que l'enseignant ait acquis une solide connaissance du domaine spécialisé, surtout lorsqu'il intervient en formation initiale, et ce, pour plusieurs raisons :

- les contenus disciplinaires sont en cours de construction chez l'apprenant. L'enseignant ne peut donc pas s'appuyer sur eux ;

- les supports ne peuvent plus être utilisés à des fins de vulgarisation à ce stade. Il serait bon que les contenus correspondent à ceux qui sont enseignés parallèlement dans le cursus afin que l'apport (input) soit compréhensible et affectivement marqué (Krashen 1981) et qu'ils soient corrélés au niveau d'acquisition des apprenants (Pienemann 1984). L'enseignant a donc généralement plus de difficultés à en comprendre le fond très « technique » et à en maîtriser la forme (le format IMRAD par exemple);

- parce que l'apprenant est investi dans son cursus et que l'enseignementapprentissage de la langue de spécialité y est généralement minoritaire, le degré de motivation est faible. En proposant un travail à la fois sur la langue et sur les contenus, on augmente le désir et l'intérêt de l'apprenant pour la langue de spécialité (Mémet 2003) ;

- la connaissance du domaine spécialisé permet d'en détourner les outils pédagogiques pour l'enseignement-apprentissage de la langue.

Qu'il soit ou non locuteur natif de la langue enseignée, l'enseignant de langue(s) a généralement suivi une formation plutôt axée sur l'acquisition de la langue générale à partir d'enseignements de linguistique, phonétique, phonologie, littérature et

\footnotetext{
${ }^{7}$ Introduction, Material (and Method), Results and Discussion.
} 
civilisation. En revanche, rares sont les formations en langues qui intègrent la langue de spécialité, et aucune formation ne propose un enseignement de la discipline. Pourtant, préconiser un enseignement de la discipline n'est pas une idée totalement nouvelle. En 2001, John Humbley écrivait :

Il s'ensuit que le professeur de langue de spécialité qui souhaite intégrer la terminologie dans sa démarche pédagogique doit impérativement se former dans la discipline dans laquelle il enseigne. [...] Mais la terminologie est en même temps le moyen qui permet à l'enseignant d'intégrer rapidement les connaissances requises pour être performant. Car posséder un minimum de connaissances ne signifie pas posséder les compétences requises pour exercer la profession en question (2001: 52).

S'il ne s'agit pas de transformer l'enseignant de langue(s) en professionnel de la discipline, il apparait essentiel de lui donner un socle de connaissances fondamentales qui lui permettront à terme de concevoir des formations adéquates et d'exploiter des supports sans que les contenus disciplinaires posent un problème de compréhension.

Cependant, il est vrai que la multiplicité des langues de spécialité pour lesquelles il existe des demandes de formation est un frein à la mise en place de formations autour d'une seule spécialité. Il nous semble donc indispensable de regrouper les langues de spécialité afin que l'enseignant puisse intervenir dans des filières sensiblement diverses mais proches.

\section{Construire des formations d'enseignants de langue(s) de spécialité}

\subsection{Développer des Masters d'enseignement et de formation}

Il n'existe, à notre connaissance, aucune formation destinée aux enseignants qui interviennent dans le milieu de la santé en France. On rencontre, en revanche, des intervenants - généralement des locuteurs natifs de la langue enseignée - issus du domaine de la santé (infirmières et médecins), notamment dans les écoles de langues privées. Malheureusement, ces enseignants n'ont pas ou peu de connaissances en didactique des langues. Il nous paraît donc indispensable de développer chez l'enseignant de langue de spécialité une double compétence à partir de formations diplômantes.

La création des Écoles supérieures du professorat et de l'éducation (ESPE) et la mise en place des nouveaux Masters "métiers de l'enseignement et de la formation » (MEF) sont l'occasion de proposer des Masters orientés vers l'enseignement des langues de spécialité.

Ainsi, à la rentrée 2014, une option «français pour la santé » est proposée dans le cadre du Master 2 «langue française appliquée » de l'UFR de langue française de l'université Paris Sorbonne conjointement avec la Faculté de médecine Pierre et Marie Curie. 


\subsection{Intégrer un enseignement du domaine spécialisé}

La plupart des enseignants ne connaissent pas ou peu le domaine spécialisé avant de prendre leurs fonctions. Pour tenter de pallier ce manque de formation spécifique, il serait bon d'intégrer un enseignement de ce domaine. Cet enseignement dispensé par des spécialistes et professionnels du domaine permettrait de donner un socle de connaissances fondamentales aux enseignants de langue(s) de spécialité.

Ainsi, dans l'option du Master 2 mentionnée ci-dessus, les étudiants reçoivent 18 heures de cours sur les six grands appareils : respiratoire, cardio-vasculaire, neurologique, gastro-intestinal, génito-urinaire et locomoteur, à partir d'une initiation à l'anatomie, la physiologie, la sémiologie et la pathologie.

Le programme comprend également un enseignement de la didactique du français médical, de la sociologie médicale (le système de santé, les établissements de soin, la filière du médicament, etc.), de la terminologie médicale (les racines gréco-latines, les néologismes, les métaphores, etc.) et de la communication en santé (la consultation, l'article de recherche, l'observation, l'ordonnance, etc.). Le stage du deuxième semestre est organisé au sein des différents services hospitaliers afin que les stagiaires puissent effectuer leurs observations dans un milieu professionnel authentique.

\subsection{Intégrer une éducation aux cultures professionnelles}

La dimension culturelle professionnelle est une dimension indispensable à l'enseignement-apprentissage d'une langue de spécialité dans la mesure où l'objectif final reste la maîtrise de savoir-être et de savoir-interagir dans un contexte professionnel. Or, les cultures sont « des notions dynamiques dont il est difficile de rendre compte sans sombrer dans la fossilisation ou le réductionnisme » (AbdallahPretceille 2013 : 10). Pourtant, offrir à l'apprenant un décryptage des usages sociaux et communicationnels afin qu'il évite les écueils liés à une méconnaissance des us et coutumes professionnels nous semble important.

Qu'il s'agisse de s'adapter au cadre professionnel stricto sensu (par exemple, une entreprise ou un hôpital) ou de communiquer avec ses acteurs (par exemple, des clients ou des patients), il est intéressant que l'apprenant puisse maitriser les codes avant d'interagir, ce qui devrait permettre un gain de temps et éviter d'éventuels échecs. C'est souvent à l'enseignant de langue(s) de spécialité que revient la charge de transmettre ces savoirs. Or, ce dernier n'est ni un professionnel du domaine spécialisé ni un ethnologue. Par conséquent, il serait bon d'intégrer, à partir d'une analyse d'échanges entre des acteurs du milieu-cible et d'entretiens avec des professionnels du domaine spécialisé, un enseignement des cultures, qui mettrait en évidence les similarités et les différences dans une démarche herméneutique et interactionniste. L'intégration de ces savoirs, qui évoluent en fonction des contextes politiques et sociaux, fait partie du processus de professionnalisation de la formation des enseignants de langue(s) de spécialité. 


\subsection{Proposer des formations plurilingues et pluridisciplinaires}

Parce que les enseignants de langue(s) de spécialité sont souvent conduits à intervenir dans plusieurs filières, il est important d'élargir leurs domaines de compétences. Nous pourrions donc envisager, à terme, la création de Masters tels que «Enseigner l'anglais et le français dans les domaines de la santé et des sciences $»^{8}$, qui comprendraient au moins deux langues et plusieurs spécialités telles que la médecine, la physique, la chimie, la géologie, la biologie, les mathématiques et l'informatique. Il serait bon de cibler des spécialités apparentées telles que, par exemple "géologie et biologie » pour les enseignants appelés à intervenir dans les UFR Sciences de la Vie et de la Terre. Une dimension plurilingue permettrait aux futurs enseignants (locuteurs natifs ou non d'une des langues proposées) d'enseigner en-dehors du cadre national (Kelly 2004).

\section{Conclusion - Pour une spécialisation des enseignants de langue(s) de spécialité}

Afin de souligner la complexité d'une langue de spécialité, nous avons rappelé quelques-unes des caractéristiques lexicales (abréviations, acronymes, toponymes, orthonymes et phraséologies) de la langue de la médecine après l'avoir replacée dans une perspective historique. Nous avons insisté sur l'importance des cultures professionnelles qu'une langue de spécialité véhicule à partir d'illustrations empruntées au domaine de la santé. Nous avons repris et développé les grandes étapes liminaires à la construction de formations en langue(s) de spécialité en nous basant sur notre expérience d'enseignante d'anglais et de français médical et en nommant les modèles d'apprentissage que nous avons retenus. Les compétences en ingénierie de formation que sous-tendent la conception et la mise en place de ces programmes nous ont conduite à remettre en question le caractère très généraliste de la formation actuelle des enseignants de langue et à préconiser une professionnalisation de cette formation afin qu'elle coïncide avec la demande croissante en LANSAD en proposant un socle de connaissances fondamentales du domaine spécialisé dans une approche plurilingue et pluriculturelle.

Cependant, nous avons souligné que cette idée se heurtait à deux difficultés. Tout d'abord, nous avons objecté qu'il était assez courant pour un enseignant en LANSAD d'intervenir dans plusieurs filières. Nous avons donc proposé de regrouper les disciplines par parenté. Par ailleurs, nous avons rappelé que les enseignants de langue qui candidatent sur des postes en LANSAD étaient très majoritairement issus d'une filière générale. Par conséquent, nous avons indiqué qu'il faudrait que le choix d'enseigner une langue de spécialité se fasse plus tôt, d'où l'intérêt qu'il y aurait à développer des options «langue(s) de spécialité » dans le cadre des MEF, voire, à créer une option « langue(s) de spécialité » au CAPES et à l'Agrégation, et à continuer de promouvoir la recherche dans ce(s) domaine(s).

\footnotetext{
${ }^{8}$ Un projet proposé dans le cadre du prochain plan quinquennal à l'université Pierre et Marie Curie.
} 


\section{Références bibliographiques}

Abdallah-Pretceille, M. 2013. L'éducation interculturelle. Paris : PUF.

Bandura, A. 1977. Social Learning Theory. Englewood Cliffs, NJ : Prentice-Hall.

Chambers F. 1980. «A re-evaluation of needs analysis in ESP ». The ESP Journal, $1(1): 25-33$.

Charpy, J.-P. 2010. «FASP médicale et substrat professionnel : le miroir éclaté ». $A S p, N^{\circ} 57: 49-65$.

Dachez, R. 2012. Histoire de la médecine: de l'Antiquité à nos jours. Paris : Tallandier

Davis, M. \& R. Harden. 1999. «Problem-based learning: a practical guide ». Med Teacher, $21: 130-40$.

Dervin, F. 2012. Impostures interculturelles. Paris : L'Harmattan.

Dudley-Evans, T. \& M. St John. 1998. Developments in English for Specific Purposes: A multidisciplinary approach. Cambridge: Cambridge University Press.

Ellis, R. 2003. Task-based Language Learning and Teaching. New York : Oxford Applied Linguistics.

Faure, P. 2012a. «Maux et mots ou la dénomination des maladies: Étude comparative anglais/français ». Neologica, numéro 6, 2012 : 191-207.

Faure, P. 2012b. L'anglais médical et le français médical: analyse linguisticoculturelle et modélisations didactiques. Paris: Éditions des Archives Contemporaines.

Faure, P. 2012c. «Enseigner une langue médicale: quelle(s) formation(s) pour quelle(s) compétence(s)?». Actes du colloque FICEL «Formation et professionnalisation des enseignants ». CIEP, novembre 2011 : 113-121.

Faure, P. 2000. «Pour une pédagogie de l'humour en didactique des langues ». Les Cahiers de l'APLIUT, Volume XIX, $N^{\circ} 4$ : 48-61.

Galanti, G.-A. 2008. Caring for Patients from Different Cultures. Philadelphia : Penne.

Giordan, A. 1998. Apprendre! Paris : Belin.

Graham, P. 2011. "Patients as team members: opportunities, challenges and paradoxes of including patients in multi-professional healthcare teams ». Sociology of Health \& Illness, Volume 33, Issue 7 : 1050-1065.

Humbley, J. 2001. «La terminologie dans les langues de spécialité ». In Memet, M. et M. Petit (eds.), L'anglais de spécialité en France. Mélanges en l'honneur de Michel Perrin. Collection ASP - GERAS Éditeur. Université Victor-Ségalen Bordeaux $2: 47-53$.

Hutchinson, T \& A. Waters. 1987. English for Specific Purposes: A learning centered Approach. Cambridge : Cambridge University Press.

Josset, P. 2010. La salle de garde : histoire et signification des rituels des salles de garde du Moyen Age à nos jours. Paris : Le Léopard d'or.

Kelly, M. 2004. Profil européen pour la formation des enseignants de langues étrangères - un cadre de référence. Rapport final, Direction générale de l'éducation et de la culture de la Commission européenne. 
Krashen, S. 1981. Second Language Acquisition and Second Language Learning. Oxford : Pergamon.

Lerat, P. 1995. Les langues spécialisées. Paris : PUF.

Mangiante, J.-M. \& C. Parpette. 2004. Le français sur objectif spécifique : de l'analyse des besoins à l'élaboration d'un cours. Paris : Hachette.

Mémet, M. 2003. «L'enseignement à contenu intégré augmente la motivation pour l'apprentissage de la langue : Vrai ou faux ? Étude portant sur des cours d'anglais de spécialité en médiation culturelle ». ASp, 39-40:131-142.

Misra-Hebert, A. 2012. « Overcoming health care disparities via better cross-cultural communication and health literacy ». Cleveland Medical Journal of Medicine, vol. 79, $2: 127-133$.

Mourlhon-Dallies, F. 2008. Enseigner une langue à des fins professionnelles. Paris : Didier.

Ofri, D. 2013. What Doctors Feel: How Emotions Affect the Practice of Medicine. Boston : Beacon Press.

Pienemann, M. 1984. «Psychological constraints on the teachability of languages ». Studies in Second Language Acquisition, 6, $2: 186-214$.

Todorov, T. 1981. Mikhaïl Bakhtine, le principe dialogique. Paris : Seuil

West, R. 1994. «State of the art article - needs analysis in language teaching ». Language Teaching, $27: 1-19$.

Weston, M.J. 2010. « Strategies for enhancing autonomy and control over nursing practice ». The Online Journal of Issues in Nursing, Vol. 15, No. 1.

http://www.medscape.com/viewarticle/723410. Consulté le 24/01/2014.

Widdowson, H. 1983. Learning Purpose and Language Use. Oxford: Oxford University Press.

Zarate, G., A. Gohard-Radenkovic, D. Lussier \& H. Penz. 2003. Médiation culturelle et didactique des langues. Strasbourg: CELV.

Pascaline Faure est Maître de conférences HDR et Directrice du Département d'anglais médical de la Faculté de médecine Pierre et Marie Curie. Elle s'intéresse principalement à la linguistique (diachronique et comparative) des langues de la médecine et aux applications qui peuvent en être faites en didactique. Ses recherches les plus récentes portent sur la formation des futurs enseignants de langues appelés à intervenir dans les milieux de la santé et sur la transdisciplinarité comme composante fondamentale dans l'acquisition et la formation en langues de spécialité. Elle travaille également sur la terminologie et, plus particulièrement sur les néologismes, les acronymes et les métaphores en français et en anglais de la médecine. Elle est membre de l'équipe de recherche DILTEC (Didactique des Langues, des Textes et des Cultures). Elle possède une double compétence : elle a été reçue au concours de PCEM1 (Premier cycle des études médicales) et a suivi des cours jusqu'en troisième année de médecine de 1995 à 1998, afin de mener à bien sa thèse d'anglais de spécialité, qui consiste en une réécriture de la grammaire de l'anglais à partir de métaphores biomédicales et à la lumière de la théorie métaopérationnelle.

pascaline.faure@upmc.fr 
